\title{
Neuroendocrine Crosstalk of Immunity
}

\section{Anil Shanker*}

Laboratory of Lymphocyte Function, Department of Biochemistry and Cancer Biology, School of Medicine, Meharry Medical College, Nashville, TN 37208, Department of Cancer Biology, Vanderbilt-Ingram Cancer Center, Vanderbilt University, Nashville, TN 37232, USA

Keywords: Immune system; nervous system; endocrine system; thymus; inflammatory reflex; infection; cancer; lymphocytes; signaling; functional cooperativity.

A wide variety of diseases and disorders ranging from Alzheimer's and cardiovascular disease to cancer are associated with the cryptic nature of inflammation and the strength of immune responsiveness. Despite impressive progress in the field of immunology during the last century, the exact triggers and magnitude controllers that regulate the dynamics of immune functions and their regulatory networks are far from being completely understood. An emerging body of evidence lends strong support that immune system interacts with the nervous and endocrine systems and the three follow overlapping mechanisms of gene regulation, signaling, cell communication and supracellular organization. In fact, the central nervous system, peripheral nervous system, the endocrine system and the immune system together form an interactive cross-system network [1]. Such a cross-system interaction adds to the already complex and vast heterogeneity and plasticity of immune cells.

This brings to the fore a burning need to expose the mechanistic affinities and cross-signaling in biological systems. It is essential to unravel the underlying fundamental principles that govern the functional organization of such complex cellular systems. Recent technological progress offers a tremendous opportunity to undertake such an effort. Present day genomic and proteomic tools and the global profiling of cellular states in terms of gene and protein expression allow an unbiased reconstruction of the genetic and molecular networks and the regulatory cross-system signaling. Cells, molecules and the organs of the immune system provide a particularly useful model for applying such approaches.

One exquisite example is the thymus that simultaneously possesses nervous, endocrine and immune functions. A great deal of data indicates that hypothalamic-pituitary axis plays a critical role in the control of thymus physiology and function [2]. In addition to being the T cell development center, thymus functions as a sensor or 'rheostat', regulating immunological homeostasis in the organism [3]. Pioneering work of Selye in 1936 showed that various physiologic and pathologic stress stimuli led to adrenal enlargement and the involution of thymus [4]. Studies have also shown that progressive growth of tumor causes thymic atrophy $[5-6,3]$, which could be reversed with the exogenous administration of thymic hormones [7-8]. These observations suggest a role of thymic endocrine function in the control of tumor growth. Thymocyte-intrinsic genetic factors also influence the selection of a tumor-reactive TCR [9] and tumor resistance [10]. A link between hormones and cancer is well known since 1896 when George Beatson discovered that surgical removal of the ovaries slows the growth of breast tumors. Thus, it would be helpful to dissect the connectivity of thymic and other endocrine mechanisms in relation to tumor growth and rejection. Tumor cells also show chemotaxis towards neurotransmitters such as dopamine and catecholamine [11-12]. There are signs of direct interactions of tumor cells with nerve cells forming a neuro-neoplastic synapse [13]. Tumors may also initiate their own innervation by the release of neurotrophic factors including the nerve growth factor and the brain-derived growth factor. Further studies in these directions will provide insights for novel strategies to fight the pathophysiology of cancer.

Chemokines and their receptors are molecular entities common to the immune and nervous systems. They present a prime example of a bidirectional communication system [14]. Originally, chemokines were found to be produced by the immune cells and thought to be completely devoted to assisting the development and function of the immune system. However, we now know that they are also produced by cells of the nervous system, such as microglia, astrocytes and neurons, regulating various aspects of the development of neural cortex and its function [15].

Immune cells are also capable of secreting neurotransmitters as well as expressing their receptors [16]. To fight pathogens, phagocytic macrophages - the front line defence cells - are transformed into proinflammatory cells that secrete catecholamines. Along with cytokines, catecholamines contribute to regulating the intensity of the immune response at the site of acute infection [17]. Recently, alternatively activated macrophages in the IL-4/IL-13-rich anti-inflammatory form have been shown to produce catecholamine and regulate the physiological task of thermogenesis [18], not previously ascribed to the immune system. Similarly, acetylcholine-synthesizing T cells have been found to relay neural signals in a vagus nerve circuit to inhibit TNF- $a$ production in the spleen by a mechanism requiring acetylcholine signaling through the $\alpha 7$ nicotinic acetylcholine receptor expressed on cytokine-producing macrophages [19]. This reveals a new role of T cells in the production of neurotransmitter acetylcholine required to control innate immune responses.

Thus, immune cells are prone to coordination by evolutionarily ancient neural circuits that operate reflexively [20]. The nervous system is hardwired to monitor the presence of cytokines and molecular products of inflammation. Patients with rheumatoid arthritis who receive anti-TNF therapy develop significant changes in brain activity before resolution of inflammation in the affected joints [21]. Studies have shown that the fever-causing activity of the pyrogenic cytokine IL-1 requires an intact vagus nerve. Administration of IL-1 into the abdomen fails to produce fever if the vagus nerve had been cut [22] Glomus cells, specialized to detect $\mathrm{pH}$ and other chemical changes in the intestinal milieu, express IL-1 receptors [23]. IL-1 binding to glomus cells may stimulate the release of dopamine, which may then trigger afferent signaling in the vagus nerve. Consequently, cytokines

*Corresponding author: Anil Shanker, Department of Biochemistry and Cance Biology, School of Medicine, Meharry Medical College, 2005 Harold D West Basic Sciences BIdg, 1005 Dr DB Todd Jr Blvd, Nashville, TN 37208, USA, Tel: 1-615 327 6460; Fax: 1-615 327 6442; E-mail: ashanker@mmc.edu

Received December 17, 2011; Accepted December 19, 2011; Published December 20, 2011

Citation: Shanker A (2011) Neuroendocrine Crosstalk of Immunity. J Blood Lymph 1:e105. doi:10.4172/2165-7831.1000e105

Copyright: ( 2011 Shanker A. This is an open-access article distributed under the terms of the Creative Commons Attribution License, which permits unrestricted use, distribution, and reproduction in any medium, provided the original author and source are credited. 
activate afferent signals in the vagus nerve, which culminate in an efferent arc to inhibit further cytokine production. The afferent arc of the neural reflex senses injury and infection by somehow "sensing" antigen and inflammatory cytokines [20, 24-25]. This activates efferent neural circuits, including the cholinergic pathway, that modulate innate inflammatory responses. This neural inflammatory reflex may provide the acute compensatory input needed to adjust the magnitude of the functional cooperativity observed among various immune cells [26,27]. Alternatively, one may be tempted to consider that the immune system may be a specialized relay system to transmit information in real time to the nervous system about the tissue response to injury and infection.

The immune and neuroendocrine systems have thus developed an intricate network of cellular specializations and communications, which allow them to sentinel the environment, sense specific changes in physical or chemical parameters and produce responses that are appropriate for and specific to the inducing signals. It would be relevant to investigate if there are any cross-system regulatory cells that have evolved and that employ common signaling mechanisms to control immuno-neuro-endocrine functional cooperativity. It is also important to understand how the complex array of 'sensory' information received by neurons, endocrine cells or lymphocytes converges to produce specific 'motor' responses, such as muscle contraction or lymphocyte activation. Methods adapted from neuroendocrinology and immunology and the powerful tools of systems biology will provide crucial leads in these integrative studies aimed at exploring neuroendocrine cross-talk of immunity.

\section{Acknowledgement}

A Shanker is supported by funds from the U54 CA091408 grant from the National Cancer Institute to the Meharry Medical College-Vanderbilt-Ingram Cancer Center partnership in eliminating cancer disparities.

\section{References}

1. Thayer JF, Sternberg EM (2010) Neural aspects of immunomodulation: focus on the vagus nerve. Brain Behav Immun 24: 1223-1228.

2. JE, Smith EM, Meyer WJ (1985) The pituitary-adrenocortical axis and the immune system. Clin Endocrinol Metab 14: 1021-1038.

3. Shanker A (2004) Is thymus redundant after adulthood? Immunol Lett 91: 79-86.

4. Berczi I (1998) The stress concept and neuroimmunoregulation in modern biology. Ann N Y Acad Sci 851: 3-12.

5. Shanker A, Singh SM, Sodhi A (2000) Ascitic growth of a spontaneous transplantable T cell lymphoma induces thymic involution. 1. Alterations in the CD4/CD8 distribution in thymocytes. Tumour Biol 21: 288-298.

6. Shanker A, Singh SM, Sodhi A (2000) Ascitic growth of a spontaneous transplantable $\mathrm{T}$ cell lymphoma induces thymic involution. 2. Induction of apoptosis in thymocytes. Tumour Biol 21: 315-327.

7. Roy R, Singh SM, Shanker A, Sodhi A (2000) Mechanism of thymocyte apoptosis induced by serum of tumor-bearing host: the molecular events involved and their inhibition by thymosin alpha-1. Int J Immunopharmacol 22: 309-321.

8. Shanker A, Singh SM (2003) Immunopotentiation in mice bearing a spontaneous transplantable T-cell lymphoma: role of thymic extract. Neoplasma 50: 272-279

9. Shanker A, Auphan-Anezin N, Chomez P, Giraudo L, Van den Eynde B, et al. (2004) Thymocyte-intrinsic genetic factors influence CD8 T cell lineage commitment and affect selection of a tumor-reactive TCR. J Immunol 172: 5069-5077

10. Shanker A, Buferne M, Schmitt-Verhulst AM (2010) Cooperative action of CD8 T lymphocytes and natural killer cells controls tumour growth under conditions of restricted T-cell receptor diversity. Immunology 129: 41-54

11. Bastian P, Balcarek A, Altanis C, Strell C, Niggemann B, et al. (2009) The inhibitory effect of norepinephrine on the migration of ES-2 ovarian carcinoma cells involves a Rap1-dependent pathway. Cancer Lett 274: 218-224.
12. Entschladen F, Palm D, Niggemann B, Zaenker KS (2008) The cancer's nervous tooth: Considering the neuronal crosstalk within tumors. Semin Cancer Biol 18: 171-175

13. Zanker KS (2007) The neuro-neoplastic synapse: does it exist? Prog Exp Tumor Res 39: 154-161.

14. Ransohoff RM (2009) Chemokines and Chemokine Receptors: Standing at the Crossroads of Immunobiology and Neurobiology. Immunity 31: 711-721.

15. Miller FD, Gauthier AS (2007) Timing is everything: making neurons versus glia in the developing cortex. Neuron 54: 357-369.

16. Blalock JE, Smith EM (2007) Conceptual development of the immune system as a sixth sense. Brain Behav Immunity 21: 23-33.

17. Flierl MA, Rittirsch D, Nadeau BA, Chen AJ, Sarma JV, et al. (2007) Phagocytederived catecholamines enhance acute inflammatory injury. Nature 449: 721-725.

18. Nguyen KD, Qiu Y, Cui X, Goh YP, Mwangi J, et al. (2011) Alternatively activated macrophages produce catecholamines to sustain adaptive thermogenesis. Nature 480: 104-108.

19. Rosas-Ballina M, Olofsson PS, Ochani M, Valdes-Ferrer SI, Levine YA, et al (2011) Acetylcholine-synthesizing T cells relay neural signals in a vagus nerve circuit. Science 334: 98-101.

20. Tracey KJ (2009) Reflex control of immunity. Nature Rev Immunol 9: 418-428.

21. Hess A, Axmann R, Rech J, Finzel S, Heindl C, et al. (2011) Blockade of TNFalpha rapidly inhibits pain responses in the central nervous system. Proc Nat Acad Sci 108: 3731-3736.

22. Watkins LR, Goehler LE, Relton JK, Tartaglia N, Silbert L, et al. (1995) Blockade of interleukin-1 induced hyperthermia by subdiaphragmatic vagotomy: evidence for vagal mediation of immune-brain communication. Neurosci Lett 183: 27-31.

23. Goehler LE, Relton JK, Dripps D, Kiechle R, Tartaglia N, et al. (1997) Vaga paraganglia bind biotinylated interleukin-1 receptor antagonist: a possible mechanism for immune-to-brain communication. Brain Res Bull 43: 357-364.

24. Huston JM, Rosas-Ballina M, Xue XY, Dowling O, Ochani K, et al. (2009) Cholinergic Neural Signals to the Spleen Down-Regulate Leukocyte Trafficking via CD11b. J Immunol 183: 552-559.

25. Rosas-Ballina M, Tracey KJ (2009) The neurology of the immune system: neural reflexes regulate immunity. Neuron $64: 28-32$

26. Malhotra A, Shanker A (2011) NK cells: immune cross-talk and therapeutic implications. Immunotherapy 3: 1143-1166.

27. Shanker A, Marincola FM (2011) Cooperativity of adaptive and innate immunity: implications for cancer therapy. Cancer Immunol Immunother 60: 1061-1074. 